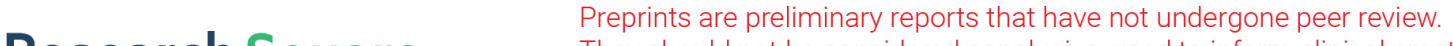 They should not be considered conclusive, used to inform clinical practice, or referenced by the media as validated information. \\ On The Bright Side of Life - The Effect of Ambient Light Intensity on Postsurgical Patient-Reported Outcomes
}

Janine Scheller ( $\square$ jasoscheller@gmail.com )

Jena University Hospital

Marcus Komann

Jena University Hospital

Claudia Weinmann

Jena University Hospital

Jonas Weinmann

Jena University Hospital

Stephan Scharnagel

Klinikum Bayreuth $\mathrm{GmbH}$

Anke Mielke

Helios Universitätsklinikum Wuppertal, Pain Clinic Wuppertal

\section{Esther Pogatzki-Zahn}

University Hospital Münster

\section{Stefan Heifeld}

Carus Dresden

Winfried Meissner

Jena University Hospital

\section{Research Article}

Keywords: postoperative pain, light exposure, nausea, patient-reported outcomes (PROMs)

Posted Date: April 30th, 2021

DOl: https://doi.org/10.21203/rs.3.rs-403129/v1

License: (c) (i) This work is licensed under a Creative Commons Attribution 4.0 International License.

Read Full License 


\section{On the bright side of life -}

2The effect of ambient light intensity on postsurgical patient-reported outcomes

3Janine Sofie Scheller ${ }^{1}$, Marcus Komann ${ }^{1}$, Claudia Weinmann ${ }^{1}$, Jonas Weinmann ${ }^{1}$, Stephan 4 Heitfeld $^{2}$, Anke Mielke $^{3}$, Esther Pogatzki-Zahn ${ }^{4}$, Stefan Scharnagel ${ }^{5}$; Winfried Meissner ${ }^{1}$

$5^{1}$ Jena University Hospital, Dept. of Anesthesiology and Intensive Care, Jena, Germany

$6^{2}$ University Hospital Carl Gustav Carus Dresden, Comprehensive Pain Center, Dresden, 7 Germany

$8^{3}$ Helios Universitätsklinikum Wuppertal, Pain Clinic Wuppertal, Wuppertal, Germany

$9^{4}$ University Hospital Münster, Dept. of Anesthesiology, Intensive Care and Pain Therapy, 10Münster, Germany

$11^{5}$ Klinikum Bayreuth $\mathrm{GmbH}$, Acute Pain Service, Bayreuth, Germany

12

13Corresponding author:

14Janine Sofie Scheller, jasoscheller@gmail.com, phone: +49 178 4537301, fax: +49 3641 159323102

16

17Disclosures:

18 - Funding: This research did not receive any specific grant from funding agencies in the

19 public, commercial, or not-for-profit sectors.

20 - Conflict of Interest: The authors declare no conflicts of interest 


\section{Abstract:}

2Light intensity affects humans in multiple ways. We aimed to characterize the potential 3impact of light intensity on patients' pain management experience in the perioperative setting. 4Within the German multicenter registry project QUIPS, we collected patient reported 5outcomes (PROs) concerning pain and side effects, demographics and perioperative pain 6medication, and measured the light intensity in their rooms on the first postoperative day. 7Primary endpoint was maximum pain intensity rated on the numerical rating scale (NRS, 0810). Secondary endpoints were pain intensity during movement, mood, nausea, tiredness 9and satisfaction. Measurement of light intensity was done with a calibrated light meter. For 10analysis, we used linear and log-linear regression models with age, gender, pre-existing 11chronic pain, ASA status, and logarithmized light intensity as independent variables. Data of 12539 surgical patients from 9 hospitals were included. We found no significant effect of light 13intensity on the primary endpoint. However, we observed a strong positive correlation 14between nausea and light intensity.

15Perspective: Our study indicates that further investigations about the clinical importance of 16light exposure with regard to nausea and other medical conditions are worthwhile.

17

18Key words:

19postoperative pain, light exposure, nausea, patient-reported outcomes (PROMs)

20

21Trial registration:

22QUIPS is registered in the German Clinical Trials Register (DRKS00006153)

23

24Ethics approval:

25QUIPS was approved by the Jena University Hospital's ethics committee (Ref.: 2722 2612/09). We confirm that all included participants signed an informed consent and the study 27was performed in accordance to the guidelines of the declaration of Helsinki. 


\section{Introduction}

2Light is defined as optical radiation entering the eye that provides visual sensation in 3humans. The effects of light fall into two categories: those modifying the individual endocrine, 4hormone, and metabolic state by light reaching the retina and those resulting from light on 5the skin. According to this specific vision related definition, light has been increasingly related 6to a range of ocular circadian, neuroendocrine, neurobehavioral and therapeutic responses 7in humans. These responses are driven primarily by stimulation of photosensitive retinal 8ganglion cells (pRGCs) that are most sensitive to short-wavelength ( app. $480 \mathrm{~nm}$ ) blue 9light[2]. Exposure to natural sunlight has been associated with improvement in mood, 10reduced mortality and decreased stress, pain and analgesic medication use and pain 11medication cost in patients who have undergone spinal surgery.[3] Among hormonal effects 12by light, the (reduced) secretion of melatonin from the pineal gland is the most important. 13Moreover, the activity of serotonin n-acetyltransferase is reduced after exposure to light. 14While light exposure elevates the serotonin levels and likewise reduces melatonin levels, 15serotonin might most likely act as a modulator of pain in the central nervous system.[4] 16Serotonin is a significant modulator of sensory input to the central nervous system; however, 17the only analgesics that selectively target G-protein-coupled 5-HT receptors are highly 18specific for treatment of headache.[4] Previous work has indicated that serotonin can both 19potentiate and inhibit primary afferent neurotransmission, and given that most subtypes of $205 \mathrm{HT}$ receptor are present in sensory ganglia, serotonin potentially has both pro- and 21antinociceptive effects through modulation of primary afferent neurotransmission.

22Postoperative pain is a major problem of modern medicine, confronting every hospital with 23structural, procedural and financial challenges. Extensive studies have demonstrated that 24many patients suffer from moderate to severe postoperative pain.[5],[6] Severe pain is 25associated with decreased patient satisfaction, delayed postoperative ambulation, increased 26incidence of chronic postoperative pain,[7] pulmonary [8], [9] and cardiac complications[10] 27and increased morbidity and mortality[11]. Options for treatment of acute, postoperative, as 
1well as for chronic pain, are limited, and many drugs are marginally effective [13] and often 2accompanied by severe side effects.

3Non-pharmacological options for the treatment of pain would be highly desirable and 4advantageous in safety, including lack of tolerance, somnolence, addiction, gastrointestinal 5disturbances and, perhaps, other factors such as convenience and cost. Light therapy has 6been reported to be useful for multiple medical conditions [14], for example to control 7depression,[15], [16] to increase daytime circadian stimulation, to improve sleep quality, and 8mitigate depression in Alzheimer disease[17]. Exposing patients to bright light of more than 96000 lux significantly improved seasonal affective disorder, a serious condition with 10increased risk of suicidality. An association has also been found between staying in a sunny 11room and a decreased need for analgesic medication following surgery. Patients who were 12accommodated on the bright side of the hospital perceived less stress and required less 13analgesic medication than those on the dark side [18]. In addition, a market for substance 14free alternatives to relieve pain emerged, including light based products like LED-belts for 15back pain treatment.

16These findings indicate a physiological relationship of pain perception and lighting condition. 17However, this relationship has not yet been studied in a larger number of patients. Therefore, 18the main objective of this study was to observe a potential association between ambient light 19intensity and pain-related patient-reported outcomes (PROs) in clinical postoperative 20routines.

21

22

\section{Methods}

\section{Study design}

25This study was based on a prospective German multicentre registry named QUIPS (Quality 26Improvement in Postoperative Pain Treatment), which was started as a benchmark initiative 27to compare pain outcome parameters among participating German hospitals. The QUIPS 28project is supported by the German Societies and Professional Associations of 
1anesthesiologists and surgeons [19]. Patients complete a validated 15-item QUIPS 2questionnaire on the first postoperative day. Patient reported data were gathered along with 3information on the type of surgery, anesthesia and pain treatment. Data assessment was 4carried out by trained study personnel. Data were collected in a standardized manner in 5randomly selected patients, unknown to the medical staff before data collection.

6Patients were informed in written form as well as orally by the study personnel that data 7collection was voluntary and anonymous and that they could refuse to be included at any 8time. Informed consent was documented by filling in the questionnaire. The project was 9approved by the Ethics Committee as well as the Data Security Board of Jena University 10Hospital, Germany, and all participating sites obtained approval from their respective Ethics 11Committees.

\section{Outcome and Influencing Variables}

13The primary outcome parameter was maximum pain intensity since surgery, measured with 14the numeric rating scale (NRS, $0=$ no pain, $10=$ worst pain imaginable). Age, gender, 15preoperative chronic pain, pain during movement and ASA-score were selected as co16variables in the analysis [20]. The secondary endpoints were mood, nausea, tiredness and 17satisfaction. These items are also part of the validated 15-item QUIPS questionnaire. The 18outcome satisfaction has been rated on a scale from 0 to $10(0=$ low grade,10 $=$ highest 19grade) while the other secondary outcome parameters nausea, tiredness etc. have been 20determined binary (yes/no).

21

\section{Patients}

23Nine hospitals participated in the study, and patients having completed the QUIPS 24questionnaire between August 2015 and September 2016 were included. Patients were 25included if the assessment was done on the day after surgery and if the patient was 18 years 26or older and able to understand the study. Exclusion criteria were the patient (1) had been 27transferred to another ward after surgery; (2) was not present in his room or had been 
1discharged at the time of data collection; (3) refused to participate in the study; (4) could not 2communicate in German; (5) had cognitive deficits or (6) was sedated or asleep.

3

\section{Light Measurement}

5The light measurement was done with calibrated light meters (unit: Ix) by a hand-held device 6(model PCE-172, manufactured by PCE Instruments, Southampton, UK) in every 7participating site. The light meter captures the luminous flux per surface unit of measure 8independent from its direction and extensiveness. The sensor has a spectral sensitivity which 9is adapted to the human photopic luminosity curve. Light intensity measurement was only 10executed on days with constant weather conditions between 9 am and $5 \mathrm{pm}$. Days with 11 frequent changes between sunshine and clouds were not included in the light study. The 12 measurement was conducted all year round to include seasonal dependencies on light 13intensity. The measurement of light intensity was performed by placing the sensor at the foot 140f the patients' bed.

15

16Statistical Analysis

17The analysis was performed using both logistic and linear regression models. We used 18logarithmic transformation in order to describe the distribution of light intensity values.

19The primary and secondary outcomes were considered as dependent variables. Age, 20 sex and preoperative pain were used as independent factors. For Integration of 21 variables into the regression model, inclusion method was applied. Furthermore we 22used the same set of covariates including age, gender, chronic pain and asa score for our 23regression models.

24A p value of $\mathbf{0 . 0 5}$ or lower was considered as significant. Data analyses were carried 25out using IBM SPSS Statistics version 24.0.

26 


\section{Results}

2Data were collected from 539 surgical patients in 9 German hospitals for 14 months from 308/15 to 09/16. Demographic (sex, age, gender) and clinical data are shown in table 1 . The 4mean worst pain intensity for all included patients since surgery was NRS of 5.7 while the 5mean light intensity measured averaged a worth of 946,76 Ix (Table 2).

6

7Patient characteristics

\begin{tabular}{|l|l|l|}
\hline age & median: 58 yrs & Q25 to $75: 55$ to 75 yrs \\
\hline gender & $54.7 \%$ female & $44.3 \%$ male \\
\hline ASA 1 & $16.3 \%$ & $\mathrm{~N}=88$ \\
\hline ASA2 & $50.3 \%$ & $\mathrm{~N}=271$ \\
\hline ASA3 & $27.5 \%$ & $\mathrm{~N}=148$ \\
\hline
\end{tabular}

8Table 1. Age, gender and American Society of Anesthesiology (ASA) state of included patients

9

10Max. pain intensity and light intensity

\begin{tabular}{|l|l|l|l|l|l|}
\hline & N & Minimum & maximum & mean & standard \\
\hline $\begin{array}{l}\text { maximum } \\
\text { pain }\end{array}$ & 537 & 0 & 10 & 5.74 & 2.73 \\
\hline $\begin{array}{l}\text { (NRS 0-10) } \\
\text { log_light }\end{array}$ & 539 & 24 & 25600 & 946.76 & 1875.59 \\
\hline Value (Ix) & & & & & \\
\hline
\end{tabular}

11Table 2. Mean values of measured maximum pain intensity and light intensity in Ix.

12 


\section{Primary Outcome maximum pain}

2

3For the primary endpoint maximum pain intensity, no significant effect of light intensity was 4found (table 3). Age $(p=0,00)$ and gender $(p=0,01)$ had a significant effect on the outcome 5 maximum pain intensity after surgery.

6Primary outcome results

\begin{tabular}{|c|c|c|c|c|}
\hline & \multicolumn{2}{|c|}{$\begin{array}{l}\text { coefficients not } \\
\text { standardized } \\
\text { univariante regression }\end{array}$} & $\begin{array}{l}\text { standardized } \\
\text { coefficents } \\
\text { multivariable regression }\end{array}$ & significance \\
\hline & $\begin{array}{l}\text { Regression } \\
\text { coefficient }\end{array}$ & $\begin{array}{l}\text { standard } \\
\text { error }\end{array}$ & Beta & \\
\hline age & -0.05 & 0.01 & -0.31 & 0.00 \\
\hline gender (male) & -0.69 & 0.24 & -0.13 & 0.01 \\
\hline chronic pain & 0.30 & 0.26 & 0.06 & 0.24 \\
\hline asa status & 0.09 & 0.27 & 0.02 & 0.66 \\
\hline Log_light intensity & -0.09 & 0.25 & -0.17 & 0.71 \\
\hline
\end{tabular}

7Table 3. Summary of statistical relation between maximum pain and age, gender, pre-existing chronic 8pain and light intensity.

9

\section{Secondary Outcome - Tiredness, Satisfaction, Pain during movement} 11

12For the secondary endpoints of pain intensity during movement, satisfaction and tiredness no 13significant effect of light intensity was found. Furthermore, the data showed a significant 14connection of gender and tiredness ( $p=0,00, \mathrm{Cl}: 1,96-4,19)$, while pain during movement 15and age ( $p=0,00, \mathrm{Cl}: 0,96-0,990)$ were statistically correlated.

16

17

18 


\section{Secondary Outcome - Nausea}

$2 A$ strong positive correlation between nausea and light intensity $(\operatorname{Exp}(B)=2,63 ; p<0.05, C l$ :

$31,62-4,26$ ) could be observed (table 4). The probability of a patient suffering from nausea 4rises by a factor of 2,6 per logarithmic light intensity value.

5 Secondary outcomes results

\begin{tabular}{|l|l|l|l|l|}
\hline & $\begin{array}{l}\text { Regression } \\
\text { coefficient }\end{array}$ & Standard error & Sig. & Exp (B) \\
\hline age & -0.03 & 0.01 & 0.71 & 0.10 \\
\hline gender (male) & 1.294 & 0.260 & 0.00 & 3.649 \\
\hline chronic pain & -0.371 & 0.251 & 0.140 & 0.690 \\
\hline ASA status & 0.165 & 0.207 & 0.424 & 1.180 \\
\hline nausea & 0.97 & 0.25 & 0.00 & 2.63 \\
\hline
\end{tabular}

6Table 4. Statistical relation between secondary outcome variables nausea, age, gender, pre-existing 7chronic pain and light intensity.

8

\section{Discussion}

10To our knowledge, this is the first analysis of an association between ambient light intensity 11and patient-reported outcome parameters related to postoperative pain management in a 12large number of patients in clinical routine. A former study evaluated the effect of sunlight on 13postoperative analgesic medication use and found that patients staying on the bright side of 14the hospital required $22 \%$ less opioid equivalent compared to those on a dark side, 15suggesting a hypothetical connection of pain perception and light intensity. [3] Thus, it could 16be suggested that pain perception after surgery and light intensity might be connected. 17However, analgesic consumption is only a surrogate of pain after surgery that can be 18affected by many different factors. Since our data show no significant relation of light intensity 19 with maximum pain perception as well as the secondary outcomes pain during movement, 
1satisfaction and tiredness, the assumption of light intensity in patient rooms having an 2influence on patients' pain perception cannot be confirmed by our observational data. 3However, regarding the differences in sample size, study conditions and methods of the two 4studies no definite comparison can be made. While the former study included only patients 5undergoing spinal surgery, calculated mean light exposure by taking measurements twice a 6day five times each from three different points of the patients' room as well as having variant 7 primary outcomes as pain medication use and cost, it is difficult to compare these two 8studies. [3]

9However, we found an unexpected positive correlation of daylight intensity and postoperative 10nausea. Up to date, there has not been any similar finding in other research concerning the 11connection of light and nausea. This finding might be explained with the metabolism of 12 melatonin [21]. The sleep hormone secreted by the pituitary gland has various functions. As 13light exposure lowers the level of melatonin via downregulation of n-acetyltransferase, higher 14concentrations of its chemical precursor serotonin could result in clinical symptoms such as 15nausea, migraine and headache, mediated via 5-HT3 and 5-HT1D receptors. Migraine 16patients are known to suffer from hypersensitivity to light following lowered excitation 17thresholds in the central nervous system [22]. In addition, a more marked melatonin 18suppression after light exposure in migraine patients compared to controls suggests that the 19melatonin level plays a central role in the pathophysiology of migraine [23]. These findings 20emphasize the assumption that photoregulation of migraine headache is exerted by the non21image-forming retinal pathway that modulates the activity of dura-sensitive thalamocortical 22neurons [24]. Finally, a recent study has shown that melatonin functioning as a biomarker for 23circadian dysregulation correlated with major depression and fibromyalgia symptom severity 24[25]. These findings manifest the hypothesis that lighting has a severe effect on 25neurohumoral pain transmission and encourage further research.

26In context to our research design that emphasizes on acute postoperative pain there is no 27further evidence suggesting a significant effect of lighting conditions on pain intensity. Our 28findings could not provide further evidence on the main hypothesis. Explanation to our 
1negative results could be the missing biological connection of the light effect organ skin and 2retina and the origin of acute pain that mainly results from structural damage and 3inflammation in the peripheral nervous system. Another reason presents itself in the delay of 4a potential effect due to the neurohumoral axis of melatonin regulation taking longer than a 5 day to have a clinical measurable impact on pain.

6The significant correlation of higher light exposure and nausea in the postoperative setting 7should be subject of further research. Thus simple implementations regarding changes of the 8postoperative lighting conditions such as dimming of the ambient light appears to provide a 9preventive option for patients that are at risk of nausea.

11Specific limitations of the study include the following: 1) The measurement of light intensity 12occurred only once at the foot of the patients' beds. This process could methodologically be 13optimized considering affected light exposure intensity due to adjusted blinds, overhead 14lighting, artificial lighting or other confounding variables. For future research, it would be 15 preferable to continuously measure light intensity variation in patient rooms during the whole 16day. 2) Due to the large patients sample size, a wide variety of different surgical patients that 17were exposed to various environmental conditions were included. 3) In the present study, 18only the room brightness (measured in lux) was determined. No difference was made 19between daylight and artificial light. Also the different color spectra of the different light 20sources and illuminants were not analyzed. Since individual colours can also cause different 21reactions, it would certainly make sense to include the distinction between daylight, artificial 22light and colour spectra of the individual artificial light sources in a further study.

23 4) As light is a complex phenomenon, having a biological impact that depends on spectrum, 24spatial distribution, timing and duration of exposure to the retina, there is a lack of valid 25 methods of measurement up to date. While the current methods including light meters have 26a spectral sensitivity adjusted to the human photopic luminosity curve, modern 27measurements should consider that the photobiologic action spectre of the greatest 28importance to humans in order to stimulate non-image forming light detection system, ranges 
1 from 290 to $270 \mathrm{~nm}$. This becomes more important if artificial light sources and lighting 2environment are involved in the process of indoor light measurement.

35) In correspondence with the results of former QUIPS analyses our data identified female 4gender, age and chronic pain and as confounders of the primary endpoint maximum pain 5regardless of the type of surgery.[20]

66) The Bonferri correction for maintaining an overall alpha-level of 0.05 was not 7implemented in our statistical analysis which could potentially raise the chance of 8false positive results.

9

10Conclusion

110ur data show no further evidence for light intensity-induced pain modulation in humans in 12the postoperative setting. For the secondary outcomes, the data showed an association 13between brighter light intensity and increased incidence of nausea for the first time.

14The subject should be studied under more controlled conditions concerning the variability of 15the types of surgery, hospital analgesic standards and potential strong differences between 16sunlight and artificial light concerning intensity and wavelength.

17The ideal study design would include measuring the light intensity average of a longer time 18span for a standardized sub group of patients undergoing similar or same surgical 19 procedures. Furthermore, in order to have less variability, only patients from one ward with 20comparable lighting conditions and architecture should be included. Randomized controlled 21trials are needed to have further evidence for making an association between light intensity 22and acute pain intensity.

23

\section{Acknowledgements}

26Much appreciation and gratitude is expressed to Antje Göttermann for coordinating the data 27collection at UKJ and to all participating hospitals for providing professional assistance 
1through study personnel and patient recruitment: Helios Klinikum Bleicherode, Helios 2Klinikum Wuppertal, Klinikum Bayreuth, Universitätsklinikum Dresden, Bezirksklinikum 3Obermain, Klinikum Orthoparc Köln, Dietrich-Bonhöffer-Klinikum Neubrandenburg, Helios 4Universitätsklinikum Wuppertal, Universitätsklinikum Münster, Universitätsklinikum Jena.

5

\section{References}

71. Meissner, W., QUIPS and PAIN OUT - present status and future perspectives. Anasthesiologie 8 \& Intensivmedizin, 2014. 55: p. 436-+.

92. Berson, D.M., F.A. Dunn, and M. Takao, Phototransduction by retinal ganglion cells that set 10 the circadian clock. Science, 2002. 295(5557): p. 1070-1073.

113. Walch, J.M., et al., The effect of sunlight on postoperative analgesic medication use: $A$ 12 prospective study of patients undergoing spinal surgery. Psychosomatic Medicine, 2005. 13 67(1): p. 156-163.

144. Connor, M., What would 5-HT do? Regional diversity of 5-HT1 receptor modulation of 15 primary afferent neurotransmission. British Journal of Pharmacology, 2012. 167(2): p. 35316355.

175. Maier, C., et al., The Quality of Pain Management in German Hospitals. Deutsches Arzteblatt 18 International, 2010. 107(36): p. 607-U14.

196. Fletcher, D., et al., A patient-based national survey on postoperative pain management in 20 France reveals significant achievements and persistent challenges. Pain, 2008. 137(2): p. 4412151.

227. Kehlet, H., T.S. Jensen, and C.J. Woolf, Persistent postsurgical pain: risk factors and 23 prevention. Lancet, 2006. 367(9522): p. 1618-25.

248. Popping, D.M., et al., Protective effects of epidural analgesia on pulmonary complications 25 after abdominal and thoracic surgery: a meta-analysis. Arch Surg, 2008. 143(10): p. 990-9; 26 discussion 1000.

279. Singh, N., et al., The effects of the type of anesthesia on outcomes of lower extremity 28 infrainguinal bypass. Journal of Vascular Surgery, 2006. 44(5): p. 964-968.

2910. Beattie, W.S., N.H. Badner, and P.T.L. Choi, Meta-analysis demonstrates statistically 30 significant reduction in postoperative myocardial infarction with the use of thoracic epidural 31 analgesia. Anesthesia and Analgesia, 2003. 97(3): p. 919-920. 
111. Rodgers, A., et al., Reduction of postoperative mortality and morbidity with epidural or spinal

2 anaesthesia: results from overview of randomised trials. Bmj-British Medical Journal, 2000.

$3 \quad 321(7275):$ p. 1493-1497.

412. Steglitz, J., J. Buscemi, and M.J. Ferguson, The future of pain research, education, and

5 treatment: a summary of the IOM report "Relieving pain in America: a blueprint for

6 transforming prevention, care, education, and research". Translational Behavioral Medicine,

$7 \quad$ 2012. 2(1): p. 6-8.

813. Finnerup, N.B., et al., Pharmacotherapy for neuropathic pain in adults: a systematic review

9 and meta-analysis. Lancet Neurology, 2015. 14(2): p. 162-173.

1014. Ibrahim, M.M., et al., Long-lasting antinociceptive effects of green light in acute and chronic 11 pain in rats. Pain, 2017. 158(2): p. 347-360.

1215. Eastman, C.I., et al., Bright light treatment of winter depression - A placebo-controlled trial.

13 Archives of General Psychiatry, 1998. 55(10): p. 883-889.

1416. Golden, R.N., et al., The efficacy of light therapy in the treatment of mood disorders: A review 15 and meta-analysis of the evidence. American Journal of Psychiatry, 2005. 162(4): p. 656-662.

1617. Figueiro, M.G., et al., Tailored lighting intervention improves measures of sleep, depression, 17 and agitation in persons with Alzheimer's disease and related dementia living in long-term 18 care facilities. Clinical Interventions in Aging, 2014. 9: p. 1527-1537.

1918. Ulrich, R.S., View through a Window May Influence Recovery from Surgery. Science, 1984. 20 224(4647): p. 420-421.

2119. Meissner, W., et al., Quality Improvement in Postoperative Pain Management Results From 22 the QUIPS Project. Deutsches Arzteblatt International, 2008. 105(50): p. 865-U8.

2320. Gerbershagen, H.J., et al., Procedure-specific Risk Factor Analysis for the Development of 24 Severe Postoperative Pain. Anesthesiology, 2014. 120(5): p. 1237-1245.

2521. al., R.H.e., The International Journal of Biochemistry \& Cell Biology 2006. 38: p. 313-316.

2622. Claustrat, B., et al., Melatonin secretion is supersensitive to light in migraine. Cephalalgia, 27 2004. 24(2): p. 128-133.

2823. Vanagaite, J., et al., Light-induced discomfort and pain in migraine. Cephalalgia, 1997. 17(7): 29 p. 733-41.

3024. Noseda, R., et al., A neural mechanism for exacerbation of headache by light. Nature 31 Neuroscience, 2010. 13(2): p. 239-U128.

3225. Caumo, W., et al., Melatonin is a biomarker of circadian dysregulation and is correlated with 33 major depression and fibromyalgia symptom severity. Journal of Pain Research, 2019. 12: $\mathrm{p}$. $34 \quad 545-556$. 
126. Rea MS, F.M., Bullough JD, Circadian photobiology: An emerging framework for lighting 2 practice and research. . Light Res Technol 2002. 34(3): p. 177-190.

3 